\title{
A distinction between the effects of sentential speaking rate and semantic congruity on word identification
}

\author{
JOANNE L. MILLER, KERRY GREEN, and TRUDE M. SCHERMER \\ Northeastern University, Boston, Massachusetts
}

\begin{abstract}
It is well known that numerous aspects of sentential context can influence the manner in which a word within the sentence is identified. We investigated two such contextual effects, that of the speaking rate of the sentence in which the target word occurs and that of the semantic congruence between the sentence and the target word. We observed that although the two effects are similar on the surface, in that each is realized as a change in the identification of acoustically ambiguous (but not unambiguous) items along a speech series, they are strikingly different in their susceptibility to changes in task demands. Specifically, changes in the task that readily eliminate the semantic congruity effect do not serve to eliminate the rate effect, suggesting that the two effects arise at different stages of analysis. The implications of this finding for models of speech processing are discussed.
\end{abstract}

It has been known for a long time that the identification of a given word in a sentence is not based solely on its own acoustic and linguistic properties, but depends as well on the acoustic and linguistic properties of the sentence in which it occurs (e.g., G. A. Miller, Heise, \& Lichten, 1951; G. A. Miller \& Isard, 1963; and see Bagley, 1900-1901, discussed in Cole \& Rudnicky, 1983). But although there is a consensus on the existence of such context effects, and continued investigation into the nature of such effects (see, e.g., Forster, 1976; Grosjean, 1980; Marslen-Wilson \& Tyler, 1980; Swinney, 1981), the manner in which sentential properties enter into the word recognition process is not yet understood.

In the course of investigations of contextual effects in speech perception, we have made an observation concerning the manner in which two different properties of a sentence influence the identification of a word within that sentence. One property is speaking rate, which is defined in terms of physical properties of the speech signal. The other is the semantic congruence between the sentence and the to-be-identified word, which is based on a linguistic, specifically semantic, analysis of the utterance. We have observed that although the two properties have superfi-

This research was supported by NINCDS Grant NS 14394, NINCDS RCDA NS 00661, HEW BRSG RR 07143, and NICHHD Contract NIH 71-2420. We thank A. M. Liberman for making available the facilities of Haskins Laboratories for preparation of the stimulus materials and P. D. Eimas for his helpful comments on an earlier version of the manuscript. Experiments 1 and 3 are the basis of a thesis by T. M. Schermer, directed by J. L. Miller, submitted for an MA degree at Northeastern University. T. M. Schermer is now at the University of Amsterdam. K. Green is now at the University of Washington. A portion of the data reported in this paper was presented at the spring meeting of the Acoustical Society of America, Chicago, 1982. Requests for reprints should be addressed to Joanne L. Miller, Department of Psychology, Northeastern University, Boston, MA 02115. cially similar effects on the identification of a word, they actually operate quite differently. The existence of the semantic effect is highly dependent on the characteristics of the task demands, whereas this appears not to be true of the rate effect. In particular, changes in the experimental task that readily eliminate the semantic effect do not serve to eliminate the rate effect. The differential sensitivity of the two effects to the experimental task suggests that the effects, although similar on the surface, arise at different stages of analysis. The purpose of the present paper is to report our observation and to discuss its implications for models of speech processing.

Our observation is based on two parallel series of studies, one of which focused on speaking rate and the other on semantic congruity. Our methodology in these studies is one that has been used extensively in speech perception research. Essentially, subjects are asked to identify members of a series of speech stimuli, in this case a series that ranged from the word "bath," which begins with the voiced stop consonant $/ b /$, to the word "path," which begins with its voiceless cognate /p/. The "bath""path" series was generated by varying the voice-onsettime (VOT) value of the initial consonant, a complex property that is known to be an important determinant of perceived voicing (cf. Lisker \& Abramson, 1970). Stimuli from the series with short VOT values are perceived as "bath," whereas those with longer VOT values are perceived as "path." And, as is typically the case with series of this type, some stimuli with intermediate VOT values are perceptually ambiguous, being heard sometimes as "bath" and sometimes as "path." In the experiments, each member of the series is presented in a sentence context that varies either in its rate of production or in its semantic content. Each of these contextual factors influences the identification of the target words, as follows. 
Consider first how sentential speaking rate would be expected to affect word identification. It is well known that speakers do not maintain a constant rate of speech when they engage in conversation (e.g., Goldman-Eisler, 1968; Grosjean \& Deschamps, 1975). One consequence of this change in rate is an alteration in the durational characteristics of acoustic properties that underlie segmental distinctions (see J. L. Miller, 1981, for a review of these findings). For example, Summerfield (1975) found that the average VOT value of syllable-initial voiceless consonants became longer as speaking rate decreased, although, interestingly, the average VOT value of voiced consonants remained relatively unchanged. With this pattern of change across rates, the VOT value that lies midway between the average VOT value of the voiced and voiceless consonants becomes longer at slower rates. This suggests that if the listener is to correctly identify voiced and voiceless consonants on the basis of VOT across a range of speaking rates, the criterion VOT value used to distinguish the two sets of consonants should become systematically longer, as speaking rate becomes slower. And, indeed, numerous perceptual experiments have shown this to be the case (e.g., Summerfield, 1981; cf. J. L. Miller, 1981). Moreover, these experiments have shown that the listener adjusts the criterion VOT value not only for the rate of the word containing the target consonant, but also for the rate of the sentence containing the target-bearing word; however, the rate of the target-bearing word itself carries the most weight and the influence of sentence rate diminishes with increasing distance from the target word (Summerfield, 1981; cf. Port \& Dalby, 1982). On the basis of these findings, we can expect that the criterion VOT value that differentiates "bath" from "path" on our series will be affected by the rate of the sentence in which it occurs: As the sentence becomes slower, the "bath""path" boundary will be located at a longer VOT value.

As for the influence of semantic context, our expectations are based on the results of a study by Garnes and Bond (1976; cf. Spencer \& Halwes, 1981). They assessed the identification of three target words, "bait," "date," and "gate," which vary in their word-initial consonant, in this case, in its place of articulation. To do so they created a "bait"-."date"'."gate" series by modifying the starting value of the second-formant transition, a property known to be important for perceived place distinctions (cf. Delattre, Liberman, \& Cooper, 1955). Each item from the series was placed in three different sentence contexts, which were semantically biased toward one of the three words. These were: "Here's the fishing gear and the ...", "Check the time and the ...", and "Paint the fence and the...". The main finding of the study was that the sentence context influenced, although did not determine, identification. That is to say, items from the series whose acoustic properties rendered them clear exemplars of one of the words were not systematically affected by the context; however, acoustically ambiguous stimuli tended to be identified as the word appropriate for the sen- tence context. In line with these results, we can expect that the location of the VOT value that distinguishes "bath" from "path" on our series will also vary as a function of sentence context: A sentence congruent only with "bath" should lead to stimuli with intermediate VOT values' being identified more often as "bath" than as "path" and, hence, to a relatively long VOT value at the "bath"-" "path" boundary, and conversely for a sentence congruent only with "path."

We will describe two sets of studies. The first established the rate and semantic congruity effects, as outlined above. The second demonstrated that these two effects are differentially sensitive to changes in the task required of the subject.

\section{ESTABLISHMENT OF SPEAKING RATE AND SEMANTIC CONGRUITY CONTEXT EFFECTS}

Two experiments were conducted. Experiment 1 established the semantic congruity effect and Experiment 2 established the rate effect.

\section{Methods}

\section{Subjects}

In each of the experiments to be described in this paper, a different group of eight student volunteers served as subjects. The students reported no history of a speech or hearing disorder and passed an audiometric screening evaluation.

\section{Stimuli}

Experiment 1-Semantic congruity effect. The stimuli for this experiment, which were edited versions of natural speech, consisted of sentences made up of a frame and a target word. Two high-frequency target words (Kučera \& Francis, 1967), "bath" and "path," were used to complete three different sentence frames. The frames were chosen on the basis of our intuitions and were intended to differ in their semantic appropriateness for the two target words. One sentence frame, "She is not thinking of the ...", is neutral with respect to the semantic properties of "bath" and "path"; it can be completed equally well with either of these words. A second frame, "She needs hot water for the ...", is semantically congruent only with "bath," whereas a third frame, "She likes to jog along the ...", is semantically congruent only with "path." All three sentence frames contain the same number of syllables and can be articulated with the same stress pattern. To corroborate our intuitions about the semantic congruence of the sentence frame and target words, we presented a written version of each sentence frame to a class of 24 undergraduate students, who were asked to complete the sentences with "bath" or "path," using the following procedure. The students were asked to assign a number on a scale from 1 to 10 to each sentence, with low numbers indicating that only "bath" was appropriate, high numbers indicating that only "path" was appropriate, and middle numbers indicating that either "bath" or "path" was appropriate. The mean ratings were $5.21(\mathrm{SD}=.51)$ for the "is not thinking" sentence, 1.33 $(\mathrm{SD}=.92)$ for the "needs hot water" sentence, and $9.88(\mathrm{SD}=.45)$ for the "likes to jog" sentence.

The stimuli were constructed in the following manner. An audio recording was made of a male speaker saying the six sentences that resulted from combining each of the three sentence frames with each of the two target words. The speaker produced each of the sentences several times at a moderate speaking rate, with sentence stress on the words "thinking," "water," and "jog." One instance of each of the six sentences was chosen for further processing. The six chosen instances were all 
articulated clearly with no hesitations or false starts and had reasonably similar overall durations, ranging from 1,150 to $1,287 \mathrm{msec}$ (excluding "the bath" or "the path"). Each of these six sentences was digitized at a 10-kHz sampling rate using the Haskins Laboratories PCM system, and an editing program was used to excise the phrase "the bath" or "the path" from each sentence at the point at which the word "the" first became audible. This resulted in six sentence frames, two for each of the three different sentences-one originally produced with "the bath" and one originally produced with "the path."

The phrases "the bath" and "the path" from the semantically neutral sentence, "She is not thinking of the bath/path", were used to construct a 16-member "the bath" "-"the path" series, in which the change from $/ \mathrm{b} /$ to $/ \mathrm{p} /$ was accomplished by manipulating the VOT value of the word-initial stop consonant using the procedure described by Ganong (1980; cf. Lisker, 1976). VOT is defined articulatorily as the time between the release of oral closure and the onset of vocal fold vibration, and is measured acoustically as the time between the abrupt increase in energy at consonantal release and the onset of periodicity. VOT is typically shorter for voiced than for voiceless stops; the VOT values of the /b/ in "the bath" and the /p/ in "the path" were 0 and $73 \mathrm{msec}$, respectively. ${ }^{1}$ The 16-member series was created by deleting successively larger acoustic segments of periodic energy from "the bath," beginning at the end of closure for $/ b /$, and replacing these acoustic segments with equally long acoustic segments of aperiodic energy taken from "the path," beginning at the end of closure for $/ \mathrm{p} /$. The durations of the successively longer acoustic segments, and hence, by definition, the VOT values of the stimuli (in milliseconds), were as follows: 7 , $12,15,19,21,26,32,35,39,45,48,53,59,63,67$, and 73 . All cuts were made at a zero crossing and those at $7,19,32,45,59$, and 73 were also located at the end of a pitch period. There was no audible indication (e.g., presence of a click) of which stimuli had been made with a cut at the end of a pitch period and which had not, nor was there any indication that the stimuli were in fact computer-edited hybrids; naive listeners consistently perceive these stimuli to be unedited tokens of natural speech. The total duration of each stimulus was $598 \mathrm{msec}$ : The duration of "the" plus closure was $180 \mathrm{msec}$ and that of "bath/path," measured from the end of closure, was $418 \mathrm{msec}$. Each of the 16 stimuli from the "the bath"-" "the path" series was joined to each of the six sentence frames (two versions of each of the three different sentences) so as to create 96 different stimuli. ${ }^{2}$

Two sets of stimulus orders were created. The first contained instances of the 16-member "the bath"-."the path" series, presented in isolation. Five different orders were recorded, each of which contained a different randomization of five tokens of each of the 16 members of the series, for a total of 80 items per order. The second set of orders contained instances of the 96 different sentences described above; each of 12 orders contained a different randomization of these sentences. If, for each of the three sentence frames, one does not distinguish between the version originally produced with "the bath" and the version originally produced with "the path," then each order contains two tokens of each of the sentence frame (3) $\times$ target word VOT (16) combinations. All stimuli were recorded with an interstimulus interval of $2.5 \mathrm{sec}$.

Experiment 2-Rate effect. The basic format of the stimulus materials was the same for this experiment as for the semantic congruity experiment, and the stimuli were prepared in essentially the same manner. The three sentence frames for the rate experiment consisted of the semantically neutral sentence frame, "She is not thinking of . ...," spoken at a fast, a medium, and a slow rate. For the medium-rate frame, we used the actual sentence frames (one originally produced with "the bath" and one originally produced with "the path") from the semantic stimulus set. To obtain the fast and slow frames, we recorded the same male talker saying this sentence, with "the bath" and with "the path," and with sentence stress on "thinking," at what he considered to be typically fast and slow rates. W/e chose, for further processing, instances of the sentence at each rate that were clearly articulated. Each of the four selected sentences was digitized as before and "the bath" or "the path" was excised. The fast, medium, and slow sentence frames had durations, averaged across the reading with "the bath" and "the path" and excluding these words, of $897,1,181$, and $1,860 \mathrm{msec}$, respectively.
For the target words, we used the same 16-member "the bath"-"the path" series that was used for the semantic studies. Each of the six sentence frames (three rates for each sentence originally produced with "the bath" and with "the path") was combined with each of the 16 members of "the bath"-"the path" series to yield a total of 96 different stimuli.

In line with the semantic experiment, two sets of orders were used in the rate experiment, isolated target word orders and sentence orders. Since the "the bath"-"the path" series was identical to that used in the semantic experiment, we used the same target word orders in the rate experiment. As for the sentence orders, we created 12 different randomizations of the 96 sentence stimuli described above (two versions of each of three sentence rates $\times 16$ target-word VOTs), and recorded these with an interstimulus interval of $2.5 \mathrm{sec}$. Again, disregarding whether the sentence had originally been produced with "the bath" or "the path," each order contains two tokens of each sentence rate (3) $\times$ target-word VOT (16) combination.

\section{Procedure}

The general procedure was the same for both experiments. Each subject participated in several sessions that were held on different days. Each session began with a warm-up phase, during which the listener identified the stimuli from one of the isolated target-word orders. The purpose of the warm-up phase was to familiarize the subjects with the target words; the data were not included in any analyses. Following the warm-up phase, the experiment proper began. During this phase, each subject was presented with a number of different sentence orders. Across the days of testing, each subject responded once to all 12 orders, thereby providing a total of 24 responses for each sentence frame $\times$ target-word VOT combination. ${ }^{3}$

During the experimental phase of each session, the subjects in both experiments were informed that they would be hearing three different sentences that ended either in the word "bath" or in the word "path" and that their task was to make two judgments about each sentence. The subjects in the semantic congruity experiment (Experiment 1) were told that they were to indicate, first, by circling BATH or PATH on an answer sheet, whether the target word was "bath" or "path" and, second, by circling on the answer sheet NEEDS HOT WATER, LIKES TO JOG, or IS NOT THINKING OF, which of the three sentences they had heard. The subjects in the rate experiment (Experiment 2) were told that their task was to indicate, first, by circling BATH or PATH on the answer sheet, whether the target word was "bath" or "path" and, second, by circling FAST, MEDIUM, or SLOW on the answer sheet, whether the sentence was uttered at a fast, medium, or slow speaking rate. In both experiments, in order to familiarize the subjects with the sentences, a randomly selected portion of one of the test orders was played before the experimental phase of the first session began.

All subjects were tested individually in a sound-treated room. The stimuli were played on a Crown SX-822 tape recorder and presented to the subjects binaurally through matched TDH-39 earphones at a comfortable listening level (approximately $78 \mathrm{~dB}$ SPL for the vowel in "bath/path"). The equipment was calibrated at the beginning of each session so that the level of presentation was kept constant across the entire experimental series.

\section{Results}

\section{Experiment 1-Semantic Congruity Effect}

Performance on the sentence identification task was, as expected, virtually perfect (greater than $99 \%$ correct). Thus, the subjects attended to the sentence context throughout the entire experiment and processed each sentence at least to the extent required to perform the task.

Performance on the target-word identification task was extremely orderly, as is apparent from Figure 1. For each sentence context, words with short VOT values were iden- 


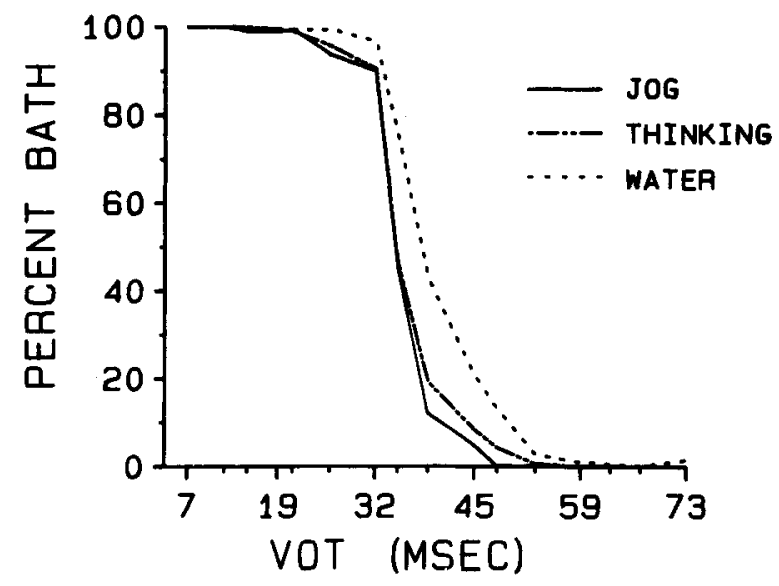

Figure 1. Mean percentage of "bath" responses as a function of the VOT value of the target word for three semantic contexts, Experiment 1.

tified primarily as "bath," whereas those with longer VOT values were identified primarily as "path," and the change from predominately "bath" to "path" responses was relatively sharp. Moreover, as predicted, the sentence frame influenced identification of items with intermediate VOT values: Compared with the neutral "is not thinking" sentence, the "needs hot water" sentence produced more "bath" responses and the "likes to jog" sentence produced more "path" responses, although the latter effect was very small. To obtain a summary measure of performance, we calculated, for each sentence context, for each subject, the VOT value at the "bath"-"path" category boundary. ${ }^{4}$ These values, which are given in Table 1, are in accord with the pattern observed in the figure. Compared with the neutral context "She is not thinking of the ...," the sentence frame "She needs hot water for the ..." led to a longer VOT boundary value, whereas the frame "'She likes to jog along the ..." had precisely the opposite effect.

The reliability of the context effect was assessed with a one-way repeated measures ANOVA on the individual boundary scores, which revealed a highly significant effect of sentence context $[F(2,14)=12.25, p<.001]$. Individual comparisons ${ }^{5}$ revealed further that the boundary value for the WATER context was significantly differ-

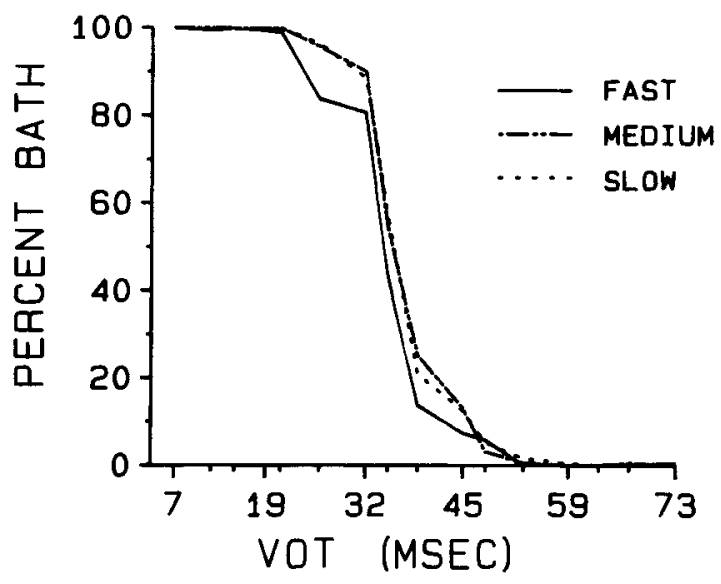

Figure 2. Mean percentage of "bath" responses as a function of the VOT value of the target word for three rate contexts, Experiment 2.

ent from that of the THINKING context $(p<.01)$ although there was no significant difference between the values for the THINKING context and the JOG context $(p>.10)$. Because we unexpectedly found a reliable context effect in only one direction, we conducted a replication experiment on a new group of listeners; precisely the same pattern of results was found. ${ }^{6}$ Thus, the asymmetry appears to be real, although the reason for it is unknown. Of greatest importance for our purposes, however, is that both studies clearly demonstrate the existence of a semantic congruity effect, if only in one direction.

\section{Experiment 2-Speaking Rate Effect}

As expected, listeners identified the rate of the sentences with very few errors (less than $1 \%$ ). Moreover, as shown in Figure 2, they identified the target words in a very orderly fashion in all three sentence contexts, with the rate of the sentence influencing the identification of items with intermediate VOT values. The mean boundary VOT values (in milliseconds) are given in Table 1. A repeated measures ANOVA on the individual boundary scores revealed that the effect of context was highly reliable $[F(2,14)=8.24, p<.01]$, and individual comparisons confirmed that the boundary values for the fast condition differed reliably from those in the medium and slow con-

Table 1

Mean Boundary VOT Value (in Milliseconds) for Target Words in Three Sentence Contexts in the Semantic Congruity Experiments (Left) and the Speaking Rate Experiments (Right)

\begin{tabular}{|c|c|c|c|c|c|c|c|c|c|c|c|}
\hline & \multirow{2}{*}{$\begin{array}{l}\text { Semantic Congruity } \\
\text { Experiments }\end{array}$} & \multicolumn{3}{|c|}{ Context } & \multirow[b]{2}{*}{ Significance } & & \multirow{2}{*}{$\begin{array}{l}\text { Speaking Rate } \\
\text { Experiments }\end{array}$} & \multicolumn{3}{|c|}{ Context } & \multirow[b]{2}{*}{ Significance } \\
\hline & & Jog & Thinking & Water & & & & Fast & Medium & Slow & \\
\hline \multirow{3}{*}{$\begin{array}{l}1 \\
3 a \\
3 b\end{array}$} & \multirow{3}{*}{$\begin{array}{l}\text { Sentence } \\
\text { Identification } \\
\text { No Sentence Identification } \\
\text { (No Speeded Response) } \\
\text { No Sentence Identification } \\
\text { (Speeded Response) }\end{array}$} & 35.2 & 35.8 & 39.6 & $\mathrm{p}<.001$ & & $\begin{array}{l}\text { Sentence } \\
\text { Identification }\end{array}$ & 34.4 & 36.7 & 36.6 & $\mathrm{p}<.01$ \\
\hline & & 31.2 & 31.4 & 32.0 & n.s. & $4 a$ & $\begin{array}{l}\text { No Sentence Identification } \\
\text { (No Speeded Response) }\end{array}$ & 34.8 & 35.6 & 36.5 & $\mathrm{p}<.02$ \\
\hline & & 36.3 & 35.9 & 36.2 & n.s. & $4 b$ & $\begin{array}{l}\text { No Sentence Identification } \\
\text { (Speeded Response) }\end{array}$ & 36.7 & 38.8 & 38.5 & $p<.05$ \\
\hline
\end{tabular}


ditions ( $p<.01$ ), which did not differ reliably from each other $(p>.10)$. Thus, there was a reliable, though small, shift in boundary location as speaking rate changed from fast to slow. We should point out that the small magnitude of the effect is not surprising, given that the rate of both the target word "bath/path" and the preceding word "the" was kept constant (see footnote 2) and, as noted above, it is known that the magnitude of the rate effect diminishes with increasing distance from the target word. For our purposes, the important point is that a change in the rate of the sentence reliably altered the identification of "bath" and "path."

\section{DEPENDENCE OF THE RATE AND SEMANTIC EFFECTS ON TASK DEMANDS}

The first set of experiments established the existence of both a semantic congruity effect and a rate effect for the identical target words. Note that in these experiments, the subject was required by the task to identify the relevant property of the sentence (semantic content or rate), as well as the target word, on each trial. We were interested in whether both effects would still obtain even when attention to the sentence was not explicity required by the task. To answer this question, we repeated the two experiments with the following procedural change: The subject was asked only to identify the target word itself on each trial-no judgment of the sentence frame was required.

\section{Methods}

Two versions of the new experiments were performed. In the first version-Experiment 3a (semantic effect) and Experiment 4a (rate effect)--the experimental procedures were identical to those of Experiments 1 and 2, with the sole exception that only one response was required on every trial; the subject was to indicate whether the target word was "bath" or "path" by circling BATH or PATH on an answer sheet. In the second version-Experiment $3 b$ (semantic effect) and Experiment $4 \mathrm{~b}$ (rate effect)-again only identification of the target word as "bath" or "path" was required on each trial. In these latter experiments, however, the subject was instructed to respond to the target item as quickly as possible by depressing the appropriately marked button (BATH or PATH) on a response panel, and was aware that his or her responses were being timed (by a small laboratory computer). There are two very different ways in which the requirement to respond quickly could influence the results. On the one hand, the speed requirement could serve to focus the subject's attention on the target word at the expense of the sentence context, thus potentially weakening the effect of context on target-word identification. On the other hand, the requirement for speed could actually lead the listener to attend to the sentence context, since it could provide information on each trial as to the precise time of occurrence of the target word and, in so doing, strengthen the effect of context.

\section{Results}

Consider first both versions of the semantic congruity experiment, Experiments 3a and 3b. As expected, in both experiments the identification of the target words was very orderly. However, in neither experiment did the semantic context influence word identification. As Table 1 shows, in each experiment the three boundary values were very similar and, in each case, a one-way repeated measures ANOVA indicated that they were not reliably different from one another $[\mathrm{F}(2,14)<1$ for each experiment]. Thus, by simply not requiring the subject to overtly identify the context sentence, we eliminated its effect on the identification of the target word, and this was true whether or not there was pressure on the subject to respond quickly.

A very different pattern emerges, however, for the two versions of the rate experiment, Experiments $4 \mathrm{a}$ and $4 \mathrm{~b}$. Not only were identification responses orderly in these two experiments, but in each case they were reliably influenced by the rate of the sentence context, as can be seen in Table 1. As for Experiment 4a, a repeated measures ANOVA on the boundary scores indicated a significant effect of context $[\mathrm{F}(2,14)=5.41, \mathrm{p}<.02]$. Individual comparisons revealed further that the fast- and slow-rate conditions differed reliably from each other $(p<.01)$, although neither differed significantly from the medium rate condition $(\mathrm{p}>.10){ }^{8}$ And, for Experiment $4 b$, a repeated measlires ANOVA also revealed a significant effect of context $[F(2,14)=3.85, p<.05]$ and individual comparisons indicated that the fast condition differed reliably from both the medium and slow conditions $(\mathrm{p}<.05)$, which did not differ significantly from each other $(p>.10)$. Thus, for the rate effect, unlike the semantic congruity effect, the elimination of the requirement to judge the sentence context did not eliminate the contextual influence on the identification of the target word, regardless of whether or not the listener was attempting to identify the target words as quickly as possible. $^{9}$

\section{GENERAL DISCUSSION}

In line with previous research, our experiments have shown that both the semantic properties of a sentence and the rate of a sentence can influence the identification of a word within that sentence. Both effects are seen as a shift in the criterion acoustic value that distinguishes acoustically similar words. And both sentential properties appear to influence only the identification of stimuli with acoustic values that are ambiguous with respect to the word to be identified: Stimuli with acoustic values that clearly specify a given word are identified as that word, irrespective of context.

The most important finding of our experiments, however, lies in the difference, rather than similarity, between the two types of context effects. Consider first the semantic effect. This effect was present only under conditions that explicitly required the subject to attend to the sentence context. This is not surprising, however, given the nature of the stimulus materials: (1) the same three sentence frames were used throughout the experiment; (2) completion of these frames with the target words resulted in incongruent sentences on approximately onethird of the trials; and (3) the subjects were fully informed of the sentence materials before the experiment began. Under these conditions, it seems reasonable for the sub- 
jects to ignore the sentence context unless explicitly required by the task to attend to it. We should point out, as well, that because of these design features, our results do not bear on the issue of the extent to which semantic context is used in word identification in a more natural situation. When processing novel sentences, for example, the listener may well use semantic context even when not required explicitly to attend to it. Clearly, a complete account of the identification process will entail a specification of the precise conditions under which semantic context does and does not have an effect. For our purposes, the important point is that at least under the present set of experimental conditions, the influence of semantic context on word identification is not obligatory.

In light of the finding that semantic context does not necessarily influence the identification of a word, it is useful to consider the stage of processing at which semantic information may have its effect when it does enter into the process. For purposes of discussion, let us assume that the subjects performed the task of identifying the words as "bath" or "path" by determining (inter alia) whether the initial consonant was $/ b /$ or $/ p /$, that is, that a segmental analysis was performed. In Experiment 1, we found that semantic context influenced the identification of acoustically ambiguous items, but not items that were clearly specified by their acoustic properties as either "bath" or "path." This indicates that the context effect was not due to a late decision process that operated on the discrete, categorical outcome of an earlier process responsible for the analysis/interpretation of the acoustic input as specifying $/ \mathrm{b} /$ or $/ \mathrm{p} /$. If that had been the case, the influence of context would have been seen across the entire series, rather than only in the boundary region. That is to say, subjects would have tended to give relatively many responses rendering the target semantically congruent with the sentence, regardless of the VOT value of the stimulus. ${ }^{10}$

Interestingly, Ganong (1980) has made precisely the same argument with respect to a related context effect, the effect of lexical status on word identification. Essentially, he showed that the identification of stimuli along a VOT series that ranged from a word to a nonword (e.g., a "dash"-_tash" series) was influenced by lexical status, such that listeners tended to identify an acoustically ambiguous item so that it constituted the word rather than the nonword; importantly, however, lexical status had much less influence on the identification of items clearly specified by the acoustic information as one of the two alternatives. On the basis of this finding, Ganong argued that lexical status cannot have its effect after a discrete phonetic decision is made (for an extended discussion of various ways in which lexical status could have its effect prior to discrete phonetic categorization, see Ganong, 1980). Thus, it appears that both the lexical status of the target item itself, and the semantic congruity between the target item and the sentence in which it occurs, have their effect at a stage of processing at which graded information from the speech signal is available. Our data indi- cate that, at least for the semantic congruity effect, there is sufficient flexibility in the processing system at this stage of analysis for the listener to base his or her judgment on the acoustic-phonetic information, without taking into account semantic context. Of considerable interest will be to determine whether this is also true of the lexical effect reported by Ganong (1980) (cf. Fox, 1984, for at least initial evidence that this may, in fact, be so).

Let us turn now to a consideration of the rate effect. What is of primary importance is that under precisely the same experimental conditions that resulted in the elimination of the semantic congruity effect, the rate effect was not eliminated. And, interestingly, a comparison of the rate effect across experiments, measured as a shift in boundary VOT value with a change in rate from fast to slow, indicated no effect of task demands on its magnitude $[F(2,21)<1]$. To be sure, the effect of rate on word identification was consistently small: The average shift in boundary VOT value between the fast and slow conditions, averaged across experiments, was approximately 2 msec. But we expected the effect to be small, given that the change in rate occurred on speech that was at least one syllable removed from the target word. Indeed, that an effect this small was reliably obtained across four experiments (including the replication) attests to its robustness.

One possibility is that the rate effect was obtained under all conditions because the adjustment for rate is an obligatory part of the word identification process. The process occurs without intent or conscious awareness [in this respect, it is like automatic processes (cf. Posner \& Snyder, 1975; Schneider \& Shiffrin, 1977)], and it is not amenable to strategic control by the subject; to use the terminology of Pylyshyn (1980; and see Fodor, 1983), the process is cognitively impenetrable. Clearly, our results provide only initial support for this conjecture. More experiments are needed that measure the rate effect under a variety of task conditions, especially those designed to eliminate, insofar as possible, processing of the context sentence. It is of interest to note in this regard, however, that in virtually all of the studies reported in the literature on the effects of sentence rate on word identification (cf. J. L. Miller, 1981), the subjects were not required by the task to attend to the sentence context. Thus, at the very least, that directed attention toward the context sentence is not required for sentential rate to influence word identification appears to be well established.

Just where in the word identification process rate has its influence is currently not known. One possibility is that a change in sentence rate affects the perceptual "clock" that is used to measure VOT as the speech signal undergoes an initial analysis (Summerfield, 1981). On this account, as speech is slowed, the perceptual "clock" runs more slowly, such that a given VOT value (near the boundary) is judged to be relatively short and, hence, is more likely to be perceived as a voiced, rather than as a voiceless, consonant. A second possibility is that a 
change in rate directly affects the placement of the criterion VOT value that differentiates voiced from voiceless consonants-as speech is slowed, the criterion is located at a longer VOT value and, hence, stimuli with intermediate VOT values are more likely to be identified as voiced than as voiceless consonants. Note that, although clearly different, both of these alternatives place the influence of rate at a relatively early stage of speech processing, namely, during the analysis/intepretation of the acoustic-phonetic information. And, interestingly, recent findings pertaining to the adjustment for rate internal to the target word indicate that the listener adjusts for the actual physical rate of the speech, rather than for its subjective rate (J. L. Miller, Aibel, \& Green, 1984), which is consistent with the view that the rate effect arises at an early stage of speech processing. ${ }^{11}$

The adjustment for rate at this early level of processing could well be a consequence of the design characteristics of the perceptual system itself (cf. Pylyshyn, 1980). If so, then rate does not have its influence because of considerable experience in listening to speech at different rates. Rather, it is an integral part of the speech perception process, presumably from the onset of functioning. It is of special interest to note in this regard that the manner in which young, prearticulate infants discriminate syllables that vary in a rate-dependent temporal property (formant transition duration) depends on the duration of the syllable, which is a concomitant of speaking rate (Eimas \& J. L. Miller, 1980). Thus, at least for rate specified within the target syllable itself, the adjustment process is operational in early infancy. ${ }^{12}$

To summarize, we have replicated the well-documented finding that the identification of a word depends in part on both the semantic content and the rate of the sentence in which it occurs. More importantly, however, we have shown that although these two contextual factors may at first glance appear to have similar consequences on word identification, they are quite different in nature: The semantic congruity effect, but not the rate effect, appears to be readily eliminated by changes in task demands. We suggest that this difference between the effects may reflect the operation of a system for which speaking rate, but not semantic content, plays an obligatory role in the processes that underlie word identification.

\section{REFERENCES}

BAGLEY, W. C. (1900-1901). The apperception of the spoken sentence: A study in the psychology of language. American Journal of Psychology, 12, 80-130.

Cole, R. A., \& RUDNicky, A. I. (1983). What's new in speech perception? The research and ideas of William Chandler Bagley, 18741946. Psychological Review, 90, 94-101.

Delattre, P. C., Liberman, A. M., \& Cooper, F. S. (1955). Acoustic loci and transitional cues for consonants. Journal of the Acoustical Society of America, 27, 769-773.

Eimas, P. D., \& MiLler, J. L. (1980). Contextual effects in infant speech perception. Science, 209, 1140-1142.

Elman, J., \& MCClelland, J. L. (in press). Exploiting lawful variability in the speech wave. Proceedings of the Conference on Invariance and Variability in Speech Processes, M.I.T.
FoDor, J. A. (1983). The modularity of mind. Cambridge: M.I.T. Press. Forster, K. I. (1976). Accessing the mental lexicon. In R. J. Wales \& E. Walker (Eds.), New approaches to language mechanisms. Amsterdam: North-Holland.

Fox, R. A. (1984). Effect of lexical status on phonetic categorization. Journal of Experimental Psychology: Human Perception \& Performance, 10, 526-540.

GANONG, W. F., III (1980). Phonetic categorization in auditory word perception. Journal of Experimental Psychology: Human Perception and Performance, 6, 110-125.

GARNES, S., \& BOND, Z. S. (1976). The relationship between semantic expectation and acoustic information. Phonologica, 285-293.

Goldman-EIsLer, F. (1968). Psycholinguistics: Experiments in spontaneous speech. New York: Academic Press.

Grosjean, F. (1980). Spoken word recognition processes and the gating paradigm. Perception \& Psychophysics, 28, 267-283.

Grosjean, F., \& Deschamps, A. (1975). Analyse contrastive des variables temporelles de l'anglais et du française: Vitesse de parole et variables composants, phénomènes d'hésitation. Phonetica, 31, 144-184.

KIRK, R. E. (1968). Experimental design: Procedures for the behavioral sciences. Belmont, CA: Wadsworth.

KLATT, D. H. (1979). Speech perception: A model of acoustic-phonetic analysis and lexical access. Journal of Phonetics, 7, 279-312.

KuČERA, H., \& FrANCIS, W. (1967). Computational analysis of presentday American English. Providence, RI: Brown University.

Liberman, A. M., \& Studdert-Kennedy, M. (1978). Phonetic perception. In R. Held, H. Leibowitz, \& H. L. Teuber (Eds.). Handbook of sensory physiology: (Vol. 8). Perception. Heidelberg: Springer.

LISKER, L. (1976). Stop voicing production: Natural outputs and synthesized inputs. Haskins Laboratories Status Report on Speech Research, SR-47, 1976.

Lisker, L., \& Abramson, A. S. (1970). The voicing dimension: Some experiments in comparative phonetics. In Proceedings of the Sixth International Congress of Phonetic Sciences, 1967, Prague: Academia.

MARSLEN-Wilson, W. D., \& TYLER, L. K. (1980). The temporal structure of spoken language understanding. Cognition, 8, 1-71.

Miller, G. A., Heise, G., \& Lichten, W. (1951). The intelligibility of speech as a function of the context of the test materials. Journal of Experimental Psychology, 41, 329-335.

Miller, G. A., \& ISARD, S. (1963). Some perceptual consequences of linguistic rules. Journal of Verbal Learning and Verbal Behavior, 2 , 217-228.

MiLleR, J. L. (1981). Effects of speaking rate on segmental distinctions. In P. D. Eimas \& J. L. Miller (Eds.), Perspectives on the study of speech. Hillsdale, NJ: Erlbaum.

Miller, J. L., Aibel, I. L., \& Green, K. (1984). On the nature of rate-dependent processing during phonetic perception. Perception \& Psychophysics, 35, 5-15.

Pisoni, D. B. (1981). In defense of segmental representations in speech processing. In Research on speech perception (Progress Report No. 7). Bloomington: Indiana University, Speech Research Laboratory.

Pisoni, D. B., Carrelt, T. D., \& Gans, S. J. (1983). Perception of the duration of rapid spectrum changes in speech and nonspeech signals. Perception \& Psychophysics, 34, 314-322.

PORT, R. F., \& DALBY, J. (1982). Consonant/vowel ratio as a cue for voicing in English. Perception \& Psychophysics, 32, 141-152.

Posner, M. I., \& SNYDER, C. R. (1975). Attention and cognitive control. In R. Solso (Ed.), Information processing and cognition. Hillsdale, NJ: Erlbaum.

Pylyshyn, Z. (1980). Computation and cognition: Issues in the foundations of cognitive science. Behavioral and Brain Sciences, 3, 111-132.

SCHNEIDER, W., \& ShIFFrin, R. M. (1977). Controlled and automatic human information processing: I. Detection, search, and attention. Psychological Review, 84, 1-66.

SPENCER, N. J., \& Halwes, T. (1981). Phonetic categorization shifts for four continua embedded in fluent semantic contexts. Journal of the Acoustical Society of America, 69, S115.

Stevens, K. N., \& Klatt, D. H. (1974). Role of formant transitions in the voiced-voiceless distinction for stops. Journal of the Acoustical Society of America, 55, 653-659. 
Summerfield, A. Q. (1975). Aerodynamics versus mechanics in the control of voicing onset in consonant-vowel syllables. In Speech perception (No. 4). Belfast: Queen's University, Department Of Psychology.

SummerField, Q. (1981). Articulatory rate and perceptual constancy in phonetic perception. Journal of Experimental Psychology: Human Perception and Performance, 7, 1074-1095.

Summerfield, A. Q., \& HagGard, M. P. (1977). On the dissociation of spectral and temporal cues to the voicing distinction in initial stop consonants. Joumal of the Acoustical Society of America, 62, 435-448.

SwINNEY, D. A. (1981). Lexical processing during sentence comprehension: Effects of higher order constraints and implications for representation. In T. Myers (Ed.), The cognitive representation of speech. Amsterdam: North-Holland.

ZUE, V. W. (1976). Acoustic characteristics of stop consonants: A controlled study. Unpublished doctoral dissertation, M.I.T. (Distributed by the Indiana University Linguistics Club.)

\section{NOTES}

1. The 0-msec VOT value for "bath" signifies that there was no discernible release burst at the consonantal release. Instead, periodicity was evident immediately after closure. The 73-msec interval of aperiodic energy for $/ p /$ included a release burst (i.e., a short period of frication) followed by a period of aspiration. The burst was very weak and was, in fact, very difficult to differentiate from the aspiration. The weak nature of the release bursts for these stimuli is in accord with the literature on the acoustic characteristics of labial consonants (e.g., Zue, 1976).

2. Two comments about our stimuli are in order. First, although VOT is often defined acoustically as a temporal interval-the interval between consonantal release and the onset of periodicity-the change from a voiced consonant (with a short VOT value) to a voiceless consonant (with long VOT value) actually entails a change in numerous properties of the signal, some of which are spectral in nature. And it has been shown that many of these properties, as well as the temporal interval itself, are perceptually relevant for the voicing distinction (e.g., Stevens \& Klatt, 1974; Summerfield \& Haggard, 1977). Because of the way in which our stimuli were constructed from natural speech, the change in the VOT interval across the speech series was accompanied by a change in many of these other properties. For example, as VOT increased, the frequency of the first formant transition at the onset of periodicity also increased. Thus, our studies investigate how context influences identification of stimuli that vary in the complex of properties associated with a change in VOT; they do not investigate how context affects identification when the voicing distinction is specified by only a single acoustic parameter. A second, related point, is that the complex property of VOT is, itself, not the only critical information for the voicing distinction. Also relevant is the duration of closure prior to consonantal release and, possibly, properties of the preceding word (in this case "the") as well. To make the comparison of the semantic congruity and rate effects in our experiments appropriate, it was especially important that the target word (series) be kept constant, and that only the sentence frame vary across contextual conditions. To ensure that this was so, we treated the word "the" as part of the target word, rather than as part of the sentence frame, thereby creating a series that ranged from "the bath" to "the path."

3. In the semantic studies, four different sentence orders were presented in each of three sessions, and in the rate studies three different orders were presented in each of four sessions. The procedural difference was due to scheduling constraints.

4. All boundary values reported in this paper were calculated by fitting a linear regression line to the data in the boundary region of the identification function (i.e., excluding the data in the tails of the functions) and taking as the boundary value the VOT value that corresponded to $50 \%$ BATH responses.

5. The individual comparisons reported in this paper were made with the least significant difference test (Kirk, 1968), using two-tailed alpha values.
6. The mean boundary values (in milliseconds) for the replication experiment were: 33.8 (JOG), 33.0 (THINKING), and 35.6 (WATER). A repeated measures ANOVA again revealed a reliable effect of context $[\mathrm{F}(2,14)=5.05, \mathrm{p}<.025]$, and individual comparisons revealed as before that the boundary values for the THINKING context and the WATER context were reliably different $(p<.02)$, whereas the values for the THINKING and JOG contexts were not $(p>.10)$.

7. It is interesting to note that the reliable change in boundary value occurred between the fast and medium and not between the medium and slow sentences, even though the change in the rate of the sentences (as measured by the difference in sentence durations) was smaller in the former case than in the latter. And this pattern of results is also seen in Experiment $4 b$ (in the other two rate experiments we report, $4 a$ and its replication, the rate effect was reliable between the fast and slow sentences; the medium sentence differed reliably from neither of these). It appears, then, that a change in sentence rate is not linearly related to a change in boundary value (cf. Summerfield, 1981, for a similar finding regarding a change in the rate of the target word itself). In order to determine what the nature of the relationship between rate and boundary value is (as well as the range of sentence rates over which a change in rate influences word identification), many sentence rates, varying from very fast to very slow, must be tested.

8. Since the rate effect was so small, though reliable, we decided to conduct a replication experiment on a new group of eight listeners. The pattern of results was identical to that of Experiment 4a. There was a significant effect of context $[F(2,14)=5.83, p<.02]$, and individual comparisons revealed a significant difference between the fast (35.9) and slow (38.7) boundary scores $(p<.01)$, but not between these and the medium (37.3) boundary score $(p>.10)$.

9. Since the reaction time data themselves are not of immediate relevance to the major concerns of this paper, they are not described. We note, however, that the patterns of reaction times in both the semantic (3b) and the rate (4b) experiments were in accord with expectations and do not affect the thrust of our arguments. For further information about these data, please write the first author.

10. Although strong arguments have been given in support of the assumption that a level of segmental representation is computed during speech processing (e.g., Liberman \& Studdert-Kennedy, 1978; Pisoni, 1981), and a number of current models of recognition incorporate such an assumption (e.g., Elman \& McClelland, in press), there is by no means consensus on this issue-in fact, it is a matter of lively debate (cf. Klatt, 1979). Thus, it is important to emphasize that the force of our discussion of the semantic effect does not depend on this assumption's being correct. Whatever the unit of representation (e.g., phonetic segment, syllable, word), our data indicate that the semantic effect does not arise at a stage of processing subsequent to a discrete classification of the input (e.g., of $/ \mathrm{b} / \mathrm{vs.} / \mathrm{p} /$ in the case of segmental units or "bath" vs. "path" in the case of word units) and, moreover, that the influence of semantic context is not a mandatory consequence of the identification process.

11. An alternative to these two accounts entails the assumption that the critical acoustic information specifying a given phonetic segment itself remains invariant under changes in rate; that is to say, timing information is intrinsic, rather than extrinsic, to the defining acoustic properties (Port \& Dalby, 1982; Summerfield, 1981). For our studies, this would mean that VOT itself is not the appropriate property to be measuring, but rather some other (as yet unidentified) property, such that, with respect to changes in this new property, the criterion value distinguishing voiced from voiceless consonants would, in fact, not change with alterations in speaking rate. That is, given that the new property would remain invariant across changes in rate, there would be no need for a separate mechanism to adjust for rate. A major problem with this account, with respect to the current finding, is that it is often assumed within an intrinsic timing framework that the relevant acoustic information for a segment will be defined precisely over that stretch of speech that is a function of the articulatory events that produce it. But in our experiments, the changing rate information occurred before the syllable that preceded the target word-surely, this lies outside of the relevant acoustic domain. It thus appears that, at least for the effect of "distant" rate information on identification, some form of an ex- 
trinsic timing acount of the rate effect will be required (cf. J. L. Miller et al., 1984).

12. There is considerable controversy over whether the mechanisms underlying rate effects-whatever their form--are specific to the processing of speech (e.g., Pisoni, Carrell, \& Gans, 1983). Although this is clearly an important issue, it should be kept in mind that a perceptual process (such as rate adjustment) can be an integral and critical part of the processes that underlie the perception of speech, without itself being specific to speech.

(Manuscript received March 13, 1984; revision accepted for publication August 2, 1984.) 